\title{
Dinámica transicional de la relación producción-inversión en el sector transporte de los estados mexicanos
}

\section{Vicente Germán-Soto*, Gloria San Gabriel Medina**}

\section{RESUMEN}

En teoría, los cambios en la composición producción-inversión evolucionan bajo dinámicas transicionales que se estabilizan en el largo plazo. Se prueba esta hipótesis mediante un modelo de Markov aplicado al sector transporte de los estados mexicanos durante 1975-2013. Los resultados señalan que se alcanza el estado estable cuando el crecimiento del producto no se condiciona a la inversión, pero resulta en una distribución desmejorada (aumentan decrecimiento y crecimiento bajo). En cambio, el escenario condicionado logra una distribución enriquecida, ya que reduce el número de episodios de crecimiento bajo y decrecimiento, eleva el crecimiento alto y mejora a las economías menos desarrolladas; sin embargo, esta dinámica de transición fluye más lentamente. El sector transporte en México requiere mayores inversiones que aceleren la transición al estado estable.

Palabras clave: infraestructura, transporte, cadenas de Markov, crecimiento económico.

Clasificación JEL: C61, H54, L91, O40.

Facultad de Economía, Universidad Autónoma de Coahuila, México. ORCID ID: https://orcid. org/0000-0001-5844-1296 Correo-e: vicentegerman@uadec.edu.mx

**Facultad de Economía, Universidad Autónoma de Coahuila, México. Correo-e: gloria_sm97@ hotmail.com 


\section{ABSTRACT}

\section{Transitional Dynamics of the Production-Investment Relationship in the Transportation Sector of the Mexican States}

In theory, changes in production-investment relationship evolve under transitional dynamics that stabilize in the long run. This hypothesis is tested using a Markov model applied to the transportation sector of the Mexican states during 1975-2013. The results indicate that the steady state is achieved when product growth is not conditioned on investment. Instead, the conditioned scenario achieves an enriched distribution, reducing the number of episodes of low growth and economic decline, raising high growth and improving less developed economies; however, this transition dynamic flows more slowly. The transportation sector in Mexico requires greater investment to accelerate the transition to the steady state.

Keywords: infrastructure, transportation, Markov chains, economic growth.

JEL Classification: C61, H54, L91, O40.

\section{INTRODUCCIÓN}

La inversión en infraestructura del sector transporte y comunicaciones constituye un revulsivo para el crecimiento económico porque implica reducciones de costos de producción y aumento de los niveles de producto. Sin embargo, también puede tener efectos negativos, cuando el desvío de recursos públicos hacia inversión en infraestructura, que busca propiciar condiciones de mayor eficiencia en la economía, no logra aumentar la producción y estimular el crecimiento. Al respecto, la OECD (2002) destaca que por lo general no se conocen a profundidad estos impactos y que esta relación debe ser investigada desde diferentes perspectivas, muestras y periodos, para contextos geográficos diversos y con producción de evidencia desde múltiples enfoques metodológicos.

¿Cómo será esta relación en el caso particular del sector transporte? ¿Habrá, como dice la teoría, una correspondencia entre tasas de crecimiento del producto y la inversión? ¿Podrá la dinámica de transición entre estas dos variables llegar a un estado estable en el sector transporte? Generalmente, se aplican enfoques que estiman el grado de dependencia entre crecimiento del producto e inversión en infraestruc- 
tura a nivel agregado, en los que juega un papel preponderante el capital público, pero no se analiza la dinámica distribucional en el tiempo que caracteriza este tipo de procesos ni se genera evidencia sobre sectores específicos de la economía.

Desde la perspectiva de economía agregada, el transporte determina y ha determinado las maneras de comprar y vender, al aumentar el mercado de los bienes y servicios producidos (Lauchlin, 1981); se relaciona con los cambios en la productividad agregada y los efectos de desplazamiento provocados por el capital público (Aschauer, 1990; Munnell, 1990; Sánchez-Robles, 1998; Dasgupta, 1999); propicia asimetrías y desigualdades regionales (Pereira y Andraz, 2006, en regiones de Portugal; Lall, 2007, en India; Ding et al., 2008, en China), aunque también se reconocen efectos positivos de largo plazo (Di Giacinto et al., 2012, en regiones de Italia).

Para Barzin et al. (2018) los impactos en la infraestructura de caminos son más importantes en países en desarrollo que en los desarrollados; además, en lugares como Gran Bretaña, los efectos son regionalmente heterogéneos (Fingleton y Szumilo, 2019), lo que da una idea de que la distancia económica en relación con el estado estacionario es un factor que contribuye a la diferenciación de resultados.

Por su parte, en México, Fuentes (2003) y Fuentes y Mendoza (2003) encontraron que la inversión en infraestructura pública puede ser un factor determinante de la desigualdad entre los estados mexicanos, ya que tiene implicaciones diferenciadas de política regional, que dependen de las características de las economías estatales. Por ejemplo, en términos de infraestructura, la región sur de México tiene una estructura económica muy distante a la del resto (Deichmann et al., 2004). Aunado a ello, Durán-Fernández y Santos (2014a) analizan el proceso de convergencia desde un concepto de productividad regional que involucra la infraestructura del transporte y anticipan que la desaparición de las brechas tomaría cerca de 25 años. Además, afirman que la infraestructura en carreteras tiene efectos positivos en el producto industrial (Durán y Santos, 2014b). Asimismo, Walke et al. (2015) encuentran un efecto-nivel desde el cual las regiones mexicanas con mayor stock de infraestructura exhiben también mayor crecimiento.

Sin embargo, la dinámica distribucional de la evolución temporal entre inversión en infraestructura y crecimiento del producto constituye un aspecto crucial poco investigado, por tanto, su valoración empí- 
rica permanece abierta en la literatura. Algunos autores, como Quah (1993), Fingleton (1997), Le Gallo (2004), han usado el enfoque de cadenas de Markov como alternativa a los análisis de corte transversal en la modelación de dinámicas del crecimiento entre regiones y como prueba condicional para comprobar efectos espaciales en la distribución del ingreso (Kang y Rey, 2018), mientras que otros, como Eslahchi y Movahedi (2012), modelan etapas de transición metodológica.

La contribución de este trabajo radica en valorar la dinámica distribucional de la inversión y el crecimiento del sector transporte mediante cadenas de Markov, es decir, como un evento probabilístico en el que el estado inicial de las inversiones lleva a un estado de crecimiento indeterminado (puede ser elevado, moderado o nulo) en el siguiente periodo, ayudando así a diagnosticar la eficacia de los programas de inversión pública para estimular el crecimiento económico. Además, dado que es un enfoque que no se basa en estimación paramétrica, no se asocia a los problemas típicos de endogeneidad, causalidad inversa o regresión espuria que se suele afrontar desde técnicas de regresión. Tampoco requiere la definición de un gran sistema de ecuaciones, como sucede cuando se usa modelación estructural (Timilsina et al., 2020), por ejemplo, los modelos tipo CGE (equilibrio computacional general). Por tanto, el enfoque de Markov proporciona una perspectiva diferente que contribuye al conocimiento empírico de esta parcela de la realidad.

Este trabajo se plantea el problema de los distintos niveles de crecimiento en cuanto a valor agregado, registrados dentro del sector transporte de las entidades federativas de México, y su dinámica de transición respecto a las variaciones en la inversión. El objetivo es investigar el proceso de transición entre diferentes estados del crecimiento para estimar la distribución que habría en el estado estable y el tiempo que llevaría alcanzar esa situación. En breve, el problema que se plantea es que en México el crecimiento económico, tanto a nivel general como sectorial, ha sido heterogéneo y volátil en tiempo y espacio, lo que puede dar lugar a que la contribución de las inversiones no sea fehacientemente apreciada. Esto es, mientras algunas entidades regionales sostuvieron tasas elevadas de aumento del producto (crecimiento elevado), otras alcanzaron tasas muy bajas (crecimiento bajo), valores cercanos a un aumento normal (crecimiento medio) o, contrariamente, tendieron a decrecer (decrecimiento). Además, estos escenarios (que podemos llamar "estados" o "situaciones") no mostraron una conducta uniforme en el tiempo. 
El estudio de la inestabilidad de la actividad económica es sumamente importante debido a que se trata de una condición en la que persisten shocks que interrumpen la función social de creación de valor; un proceso es destruido, en tanto que otro (nuevo) no se ha formado aún, lo que provoca que el orden económico no logre estabilizarse como ritmo normal de producción.

Por tanto, es de gran interés investigar si hallarse en alguna escala del crecimiento tendrá carácter permanente o transitorio, así como saber cuánto tiempo se necesita para que los movimientos de transición se estabilicen; esto es, si las condiciones actuales de movimiento transicional entre un estado y otro se mantienen, ¿cuánto tiempo llevará alcanzar un equilibrio? ¿Cómo será la dinámica transicional en regiones de mayor y menor desarrollo? La respuesta a estas interrogantes permitirá saber si una economía que registra decrecimiento (o crecimiento alto), por ejemplo, tendrá posibilidades de salir (o permanecer) y cuánto tiempo tardará, información fehaciente en la toma de decisiones de los sectores público y privado.

La hipótesis de investigación es que entre producto e inversión existe una fuerte correspondencia cuya composición evoluciona a una situación de equilibrio en el largo plazo. Esta predicción se pone a prueba en el sector transporte de los estados mexicanos durante el periodo 19752013, mediante un modelo de cadenas de Markov alimentado con las tasas de crecimiento (inversión y valor agregado) registradas por las entidades federativas del país. Sin embargo, dadas las características del desempeño de la economía mexicana, el proceso de transición entre un estado y otro podría ser muy lento; es decir, puede llevar algún tiempo. Si una economía sostiene crecimiento económico elevado o bajo, tal vez decrecimiento, tenderá a permanecer en esta situación, con pocas posibilidades de rotación. De ser complicada la mejora del desempeño en el corto plazo, es probable que el sector del transporte mexicano marche hacia una "trampa del crecimiento".

Las ideas esbozadas hasta aquí se extienden a cuatro secciones, además de la introducción y las conclusiones. Un panorama de estudios relacionados con el tema se presenta en la sección 1, mientras que el método de contrastación empírica se expone en la sección 2. La parte 3 describe y explora la base de datos; la sección 4 plasma los resultados $\mathrm{y}$, finalmente, se plantean las conclusiones principales. 


\section{REVISIÓN DE ESTUDIOS}

Una gran cantidad de trabajos sobre el crecimiento e inversión dentro del sector transporte se centra en la estimación de la dirección y magnitud provocados por sus efectos. En general, la evidencia concluye una relación positiva en el sentido de que la inversión estimula el crecimiento del sector. Una de las funciones principales del transporte es la incorporación y expansión del ámbito geográfico, ya que así se crea el desplazamiento comercial e industrial de los diversos procesos de producción y distribución.

A pesar de que hay un consenso de la importancia de las mejoras de infraestructura en el transporte, ya que agilizan el movimiento de mercancías, productos y personas y aceleran el crecimiento económico, se contabilizan muy pocos trabajos con metodologías basadas en el estudio de la dinámica de la distribución temporal y/o espacial, como el enfoque de Markov. Es posible que en este vacío influya la disponibilidad de datos para estimar procesos tipo Markov. De esta manera, la mayoría de las publicaciones se centra en técnicas paramétricas, en sus diversas modalidades (corte transversal, panel, raíces unitarias, modelos autorregresivos, etc.), en funciones de producción para medir las elasticidades o en el uso de enfoques de modelación estructural, entre otros.

En este sentido, Cohen y Morrison (2003 y 2004) estudian esta relación en los Estados Unidos de América y encuentran efectos diversos en función de las características particulares de infraestructura de cada estado. Por ejemplo, hay evidencia de efectos derrame en las entidades conectadas por la expansión aeroportuaria, similares en aquellas que poseen aeropuertos centrales, mientras que un tercer grupo concentra los mayores impactos. Respecto a la inversión en autopistas realizada de 1982 a 1996, los autores confirman la existencia de efectos espaciales. En cuanto a las entidades que realizan inversión en puertos, son mayormente beneficiadas y no se encontró un efecto expansivo entre economías físicamente contiguas.

Sobre los impactos positivos de las infraestructuras del transporte en España, Nombela (2005) aporta apoyo empírico al obtener elasticidades positivas sobre la producción desde dos metodologías y distintas fuentes de información (función Cobb-Douglas y datos de panel). No obstante, otros estudios señalan efectos adversos. Por ejemplo, Maibach 
et al. (2007) encontraron que los sistemas de transporte generan externalidades negativas, como la congestión y sus costos relacionados, entre los que destacan pérdida de tiempo, impactos ambientales, emisiones de gases de efecto invernadero, contaminación del aire y ruido; también se generan costos de salud derivados de ellos. La importancia concedida a los costos externos, cuando se trata de elegir entre prioridades políticas en competencia, aumenta junto con los ingresos de un país. Así, Khadaroo y Seetanah (2008) descubren que en las Islas Mauricio la inversión en capital del transporte ha sido un elemento determinante en el desarrollo de la economía; sin embargo, su impacto ha sido menor en comparación con otros tipos de capital.

LeSage y Polasek (2008) relajan el supuesto de homogeneidad espacial cuando consideran la existencia de interacciones entre los espacios geográficos. De acuerdo con la teoría espacial, las estimaciones y los resultados pueden variar de manera significativa si no se considera dentro del análisis regional la dependencia que resulta de la proximidad geográfica. A través de métodos econométricos espaciales y datos de 1985 a 2006, Hu y Liu (2010) hallaron efectos positivos en el transporte en China; tanto modelos teóricos como empíricos confirman efectos derrame positivos desde la inversión.

Para Di Giacinto et al. (2012) la infraestructura del transporte influye positivamente en agregados económicos como PIB y capital privado. El impacto se atribuye a las políticas coordinadas; se realza la importancia para los sectores económico y político, ya que invita a reflexionar sobre los mejores canales de coordinación que deben observarse entre las autoridades locales para que los sistemas institucionales descentralizados puedan explotar plenamente las externalidades positivas.

Para Ding (2013), a medida que aumenta la inversión en infraestructura del transporte y los precios llevan a disminuir ciertos insumos, como la mano de obra, se genera una atracción de empresas e inversiones en las grandes urbes o áreas centrales y se crean brechas crecientes entre el núcleo y la periferia.

De este modo, la inversión en infraestructura del transporte puede contribuir al crecimiento y ampliar el stock de capital disponible para su uso en la producción de bienes y servicios. Con más capital y mayor eficiencia en la producción, pueden aumentar los niveles de ingreso real y de bienestar (German-Soto y Barajas, 2014). Tal como lo muestran 
Chen y Haynes (2015), las autopistas tienen un impacto mayor en el crecimiento, el tren de pasajeros y los aeropuertos son complementarios a otros modos de transporte y, a su vez, imprimen un impacto significativo en la producción de la región noreste de los Estados Unidos.

La mayoría de los autores coinciden en una relación positiva a largo plazo. En la India, por ejemplo, se asocia más con la teoría del crecimiento endógeno y la ley de Wagner (Maparu y Mazumber, 2017). Mientras que para Barzin et al. (2018) hay efectos heterogéneos en función de si se trata de un país desarrollado o en desarrollo; también parece haber impactos diferenciados en el ámbito de estudio de las regiones de un país (Fingleton y Szumilo, 2019). Por su parte, Kyriacou et al. (2019) analizan la eficiencia administrativa de 34 países y descubren que es posible materializar objetivos de crecimiento aceptables sin que sean necesarios niveles elevados de inversión en infraestructura; igualmente, se pueden alcanzar los mayores impactos económicos en función de un cierto nivel de inversión, resultado útil dadas las restricciones presupuestarias que a menudo afrontan los países para invertir y que es crucial investigar, principalmente en países donde los recursos para el desarrollo económico tienen más limitaciones (Timilsina et al., 2020).

El impacto de las inversiones dependerá esencialmente del nivel de desarrollo del país (Chen y Haynes, 2015). Los efectos se pueden dividir en endógenos y exógenos. Los primeros son aquellos generados en la misma inversión del transporte, como la reducción del tiempo de viaje, mientras que los segundos son los que se dan en el resto de la economía, como la disminución de los precios generados gracias a los efectos endógenos, el aumento de los flujos de producción, las materias primas, etc.

Con esta breve reseña se infiere que el enfoque de Markov no tiene aún mucha presencia en los estudios sobre el papel del transporte en la economía. La mayor parte de los trabajos con este método analizan convergencia de ingresos entre conjuntos de economías (Fingleton, 1997; Bickenbach y Bode, 2003; Le Gallo, 2004; Kang y Rey, 2018); abordan dinámicas de enfermedades endémicas (Eslahchi y Movahedi, 2012); consideran el desempeño del sector salud o estudian otra clase de procesos, como los de deuda-crecimiento, evolución del mercado de la electricidad, entre otros. Por lo anterior, se entiende que el núcleo central de la técnica son los comportamientos dinámicos; sin embargo, 
es posible aprovechar sus propiedades analíticas para el entendimiento de la dinamicidad mostrada por el sector transporte, principalmente en relación con la persistencia de estados iniciales desfavorables. Por ejemplo, qué tan difícil será salir de un estado de crecimiento bajo, condicionado al nivel de inversión realizado. Se deben examinar las distintas combinaciones inversión-producción para inferir sobre la velocidad de transición.

\section{EL MODELO DE MARKOV}

Los modelos de transición de Markov consisten en combinaciones sucesivas en el tiempo de dos diferentes matrices: una de condiciones iniciales, digamos $X_{t}$, y otra que integra los movimientos de transición entre los varios estados definidos, digamos $M$, la cual constituye un cuadro ordenado de probabilidades. Se asume que la matriz $X_{t}$, que contiene el desempeño de un conjunto de economías, es un proceso estocástico finito; es decir, satisface la propiedad de Markov (Hillier y Lieberman, 2010) definida como:

$$
\mathrm{P}\left(X_{t}=j \mid X_{t-1}=i, X_{t-2}=i_{t-2}, X_{t-3}=i_{t-3}, \ldots, X_{0}=i_{0}\right)=P\left(X_{t}=j \mid X_{t-1}=i\right)
$$

donde $t \in T \forall T=0,1,2,3, \ldots$ en todos los posibles estados $j, i, i_{m}(\forall m=0,1,2,3, \ldots$, $m-2)$ por lo que la matriz de probabilidades $X$ se encuentra en el estado $J$, en el periodo $t$, y sólo depende del estado $i$ y el periodo $\mathrm{t}-1$, pero no de estados de periodos previos.

Siguiendo las investigaciones dentro de esta línea (Quah, 1993; Fingleton, 1997; Le Gallo, 2004) y el desarrollo de la técnica expuesta en varios trabajos (por ejemplo, Hillier y Lieberman, 2010), se asume una cadena de Markov estacionaria en el tiempo; es decir, las probabilidades de transición $m_{i j}(t)=P\left(X_{t}=j \mid X_{t-1}=i\right)$ son independientes, por lo que es posible tener una distribución homogénea: 


$$
M=\left[\begin{array}{llll}
m_{11} & m_{12} & \ldots & m_{1 N} \\
m_{21} & m_{22} & \ldots & m_{2 N} \\
m_{31} & m_{32} & \ldots & m_{3 N} \\
\vdots & \vdots & \ddots & \vdots \\
m_{N 1} & m_{N 2} & \ldots & m_{N N}
\end{array}\right]
$$

Además, por fila, $M$ debe sumar $1, \sum_{i=1}^{N} m_{i j}=1$, mientras que el vector inicial que describe la frecuencia con la que las regiones se hallan en cada estado definido en el tiempo de partida, se expresa mediante la siguiente ecuación:

$$
X=\left[\begin{array}{lllll}
m_{1} & m_{2} & m_{3} & \ldots & m_{N}
\end{array}\right]
$$

con $N$ igual al número de estados considerados. Las ecuaciones (2) y (3) definen las distribuciones después de algún periodo de transición y en función de ciertas condiciones, como la ley de movimiento que se determina desde la matriz $M$ y las probabilidades sobre los $N$ nodos iniciales descritos en (3). Por ejemplo, la distribución después del primer periodo de transición (en el momento $t=1$ ) se define como $X_{t=1}$ $=X_{t=0} M$ y en el $T$-ésimo periodo $X_{t=T}=X_{t=T-t} M$. Dada la característica de ser una matriz regular, la cadena de Markov tiende a una distribución límite en el tiempo $X^{*}=\lim _{T \rightarrow \infty} X_{t=0} M^{T}$ que es independiente de su asignación inicial, $X_{t=0}$.

La aplicación empírica al análisis de regiones o estados de un país es bastante sencilla. Para cada región $r$, las $m$ secuencias describen los estados en cada periodo $t$. En este trabajo, las $r$ secuencias se toman como realizaciones independientes de la cadena de Markov definida en el espacio finito de $N$ estados posibles, una matriz de probabilidades de transición $m_{i j}$ y un reparto inicial $X_{t=0}$ Además, la distribución límite $X^{*}$ es calculada para el arreglo matricial de probabilidades de transición estimadas, $M$. La comparación entre los resultados de las matrices final e inicial constituye información imprescindible sobre el tiempo que tardarían las condiciones iniciales en desaparecer y, por tanto, es informativa de la persistencia de las distintas etapas del desempeño 
económico, como crecimiento alto, moderado, negativo, etc., resultado interesante que permite, por ejemplo, valorar la dinámica de la relación temporal existente entre inversión y producción en un conjunto de economías.

Por su parte, la frecuencia de las transiciones en el periodo de estudio se obtiene desde los datos observados de las variables y a partir de la consideración del cálculo siguiente:

$$
\hat{m}_{i j}=\mathrm{f}_{i j} / \mathrm{f}_{i}=\mathrm{f}_{i j} / \sum_{j} \mathrm{f}_{i j}
$$

donde $f_{i j}$ y $f_{j}$ significan sumas de las frecuencias observadas en todos los periodos de transición, esto es, $\mathrm{f}_{i j}=\sum_{t=1}^{T} \mathrm{f}_{i j}^{t}$ y $\mathrm{f}_{i}=\sum_{t=1}^{T} \mathrm{f}_{i}^{t-1}$ (véase, por ejemplo, Hillier y Lieberman, 2010).

A pesar de su sencillez, el método no escapa de algunas restricciones. Una es que las estimaciones de las probabilidades de transición dependen de que la muestra de datos reúna las condiciones exigidas por la teoría de cadenas de Markov, requisito que no siempre se satisface dada la poca disponibilidad de información. Otra proviene de las estimaciones, ya que éstas deben apoyarse en un número de observaciones suficientemente grande, tanto de unidades de corte espacial como temporal. Lo aconsejable aquí es usar el mayor número posible de datos. Aun con ello, este enfoque presenta propiedades deseables. Por ejemplo, cuando la matriz de transición aumenta a potencias cada vez más elevadas, converge hacia la igualdad de renglones, lo que se denomina estado estable. Es decir, las probabilidades dentro de la matriz son las mismas, lo que significa que permanecer en algún estado ya no depende de la situación inicial y esta información es crucial para entender los efectos condicionados entre producción e inversión, sin las perturbaciones (o ruido) que provocan los cambios en las variables, es decir, los movimientos comunes a cualquier proceso en transición.

\section{DATOS, DEFINICIÓN DE LOS ESTADOS DE TRANSICIÓN Y ANÁLISIS EXPLORATORIO}

La base de datos se integra con información por entidad federativa de México sobre valor agregado e inversión privada del sector transporte, que es posible recoger de los censos económicos de INEGI. Esta clase 
de registros se colecta cada cinco años, de tal forma que el panel final cubre el periodo de 1975 a 2013. ${ }^{1}$ Es conveniente destacar que el sector transporte en este trabajo se constituye de información de los subsectores del autotransporte de carga (cuyo código censal es 7112), autotransporte de pasajeros (7113), aeronaves (7131) y el sector de comunicaciones (7200). Sólo unas pocas subramas, como el tren eléctrico y los ferrocarriles, no fueron consideradas por no tener representatividad a nivel estatal, o bien porque la información estadística no cubría todo el periodo de análisis.

Con los valores estadísticos de ambas variables se calculan tasas de crecimiento y se forman cuantiles que agrupan la información en tiempo y espacio, lo que da lugar a cinco clases mutuamente excluyentes. Una incursión exploratoria de la base de datos sugiere que en el sector del transporte mexicano se tienen tasas muy elevadas de crecimiento (superiores a 6\%), y en algunos otros casos, episodios de crecimiento muy bajo y tasas negativas. De esta manera, la organización de cuantiles se corresponde también con los intervalos de clase de las tasas de crecimiento reportadas en el Cuadro 1, mismos que se identifican con la siguiente nomenclatura: crecimiento muy alto (CMA), crecimiento alto $(\mathrm{CA})$, crecimiento medio $(\mathrm{CM})$, crecimiento bajo $(\mathrm{CB})$ y decrecimiento (DEC).

\section{CUADRO 1}

DEFINICIÓN Y LÍMITES DE LOS ESTADOS DEL CRECIMIENTO

\begin{tabular}{cc}
\hline Estado/Clase & \multicolumn{1}{c}{ Criterio } \\
\hline Crecimiento muy alto (CMA) & Tasas de crecimiento iguales o superiores al $6 \%$ \\
\hline Crecimiento alto (CA) & Tasas de crecimiento entre $4 \%$ y $6 \%$ \\
\hline Crecimiento medio (CM) & Tasas de crecimiento entre $2 \%$ y $4 \%$ \\
\hline Crecimiento bajo (CB) & Tasas de crecimiento entre $0 \%$ y $2 \%$ \\
\hline Decrecimiento (DEC) & Tasas de crecimiento negativas \\
\hline
\end{tabular}

Nota: los intervalos cerrados no incluyen el límite superior. Fuente: elaboración propia.

El periodo seleccionado responde a los años con información comparable y homogénea en tiempo y espacio. Los años previos a 1975 no fueron considerados debido a que la metodología de censos cambia sustancialmente. 
Para distinguir el papel de las variables que son objeto de investigación, se llevan a cabo dos tipos de experimentos. En el primero (ejercicio 1) se analiza la dinámica de transición en forma individual. La idea es examinar, para cada variable, la dinamicidad temporal definida por los diferentes estados del crecimiento, por ejemplo, si un periodo de CA se mantiene en el siguiente o es más probable que cambie de estatus.

En el ejercicio 2 se estudia la dinámica de transición al combinar la correspondencia entre ambas variables. Por ejemplo, es posible tener crecimiento alto de forma simultánea en valor agregado e inversión privada, o casos de crecimiento alto del valor agregado combinados con crecimiento bajo, o decrecimiento, en las inversiones, y así sucesivamente.

\section{RESULTADOS Y ANÁLISIS}

\subsection{El caso general de las 32 entidades federativas (muestra absoluta)}

El vector inicial se genera al contabilizar el número de eventos ocurridos en cada estado/clase durante el periodo de estudio (Cuadro 2). Los cuadros 3,4 y 5 muestran la combinación de probabilidades de transición, así como la distribución final en el estado estable, para ambos ejercicios empíricos. Estas probabilidades se calculan a partir de los movimientos relativos que las entidades federativas registraron, en términos de crecimiento, en el transcurso del periodo de estudio de este trabajo; es decir, la frecuencia relativa de estar en cada uno de los estados previamente definidos. En el Cuadro 5, correspondiente al ejercicio 2, las clases del crecimiento para el valor agregado se especifican por fila, mientras que las de inversión se detallan por columna. 
CUADRO 2

VECTOR DE VALORES DE LA DISTRIBUCIÓN INICIAL

\begin{tabular}{cccccc}
\hline \multicolumn{5}{c}{ Ejercicio 1: tasas de crecimiento no condicionadas (por variable) } \\
Estado & CMA & CA & CM & CB & DEC \\
Eventos (valor agregado) & 62 & 10 & 17 & 14 & 122 \\
\hline Eventos (inversión privada) & 83 & 8 & 7 & 19 & 107 \\
& Ejercicio 2: tasas de crecimiento condicionadas & & \\
Estado & CMA_INF & CA_INF & CM_INF & CB_INF & DEC_INF \\
\hline Eventos & 70 & 10 & 19 & 18 & 139 \\
\hline
\end{tabular}

Nota: se usa la extensión INF para las tasas de crecimiento de la inversión privada; para el resto de las variables véase el Cuadro 1.

Fuente: elaboración propia.

Un análisis exploratorio de la información contenida en los cuadros 3, 4 y 5 sugiere que en México los procesos de crecimiento e inversión privada del transporte han presentado cierta volatilidad, ya que los valores más altos de probabilidad, así como el número de eventos (Cuadro 2), se concentran en los cuantiles extremos. La probabilidad de estar en alguno de los estados definidos y permanecer allí un periodo después es relativamente baja, excepto para la clase "decrecimiento", la cual estima un valor de 43\% (valor agregado), 35\% (inversión) y $62 \%$ (ejercicio condicionado), cifras que aluden a lo difícil de salir de esa situación desfavorable. Además, durante el periodo de estudio, la probabilidad de estar en "decrecimiento" fue muy elevada y superior a las situaciones restantes. En el estado estable también resulta ser la clase que reporta las probabilidades más altas en casi todos los ejercicios. Excepciones son, como se puede ver, el examen condicionado, mismo que deja una mejor distribución.

Al registrarse las probabilidades más elevadas en las categorías crecimiento muy alto (CMA) y decrecimiento (DEC), se forma una dinámica de transición concentrada en los extremos (volatilidad); se crece muy alto en algún periodo, o bien se decrece, pero la dinamicidad es baja en las posiciones intermedias, lo que se relaciona con elevada volatilidad. Este desempeño es confirmado por los valores del estado inicial reportados en el Cuadro 2, donde se observa que la mayor parte de los eventos ocurren con crecimiento muy alto (CMA) o con decrecimiento (DEC), lo que nuevamente sugiere elevada volatilidad. ¿Qué tan fuerte es esta aparente conducta volátil? 
En el Cuadro 3 se observa que, partiendo del estado y matriz de probabilidades iniciales, la estabilidad se alcanza en 11 periodos. Este número indica el tiempo necesario para que desparezcan las condiciones iniciales. Tras la estabilización de la matriz de transición hay un aumento en el número de episodios que promedian decrecimiento, mientras se reducen ligeramente aquellos que exhiben crecimiento muy alto. El resto de los estados se mantiene casi invariable, excepto crecimiento bajo, el cual presenta una leve tendencia a subir. Esto significa que las condiciones de crecimiento del valor agregado del sector transporte no tienen mucha probabilidad de mejorar, pero sí de empeorar.

CUADRO 3

MATRIZ DE TRANSICIÓN Y ESTADO ESTABLE DEL CRECIMIENTO DEL VALOR AGREGADO

\begin{tabular}{ccccccc}
\hline & CMA & CA & CM & CB & DEC & VPEE \\
CMA & 0.113 & 0.048 & 0.048 & 0.097 & 0.694 & 0.2586 \\
CA & 0.000 & 0.000 & 0.100 & 0.000 & 0.900 & 0.0479 \\
CM & 0.000 & 0.118 & 0.118 & 0.118 & 0.647 & 0.0619 \\
CB & 0.071 & 0.071 & 0.071 & 0.000 & 0.786 & 0.0735 \\
DEC & 0.402 & 0.041 & 0.057 & 0.074 & 0.426 & 0.5581 \\
VD & 59 & 11 & 14 & 17 & 126 & EE $=11$ \\
\hline
\end{tabular}

Nota: $\mathrm{VD}_{\mathrm{EE}}=$ vector de distribución en el estado estable, $\mathrm{VP}_{\mathrm{EE}}=$ vector de probabilidades del estado estable $\mathrm{y}$ $\mathrm{EE}=$ periodos necesarios para alcanzar el estado estable; para el resto de las variables véase el Cuadro 1. Fuente: elaboración propia.

Por su parte, la inversión privada requiere mayor tiempo para estabilizarse (Cuadro 4) y deja una situación de equilibrio menos satisfactoria. Hay mayor acentuación de los puntos extremos; es decir, se reduce el número de casos con crecimiento muy alto (83 versus 66 ) y se incrementan los episodios con decrecimiento (107 versus 115).

Los resultados cambian notablemente cuando se lleva a cabo el ejercicio 2 (Cuadro 5). En teoría, el crecimiento del producto se encuentra condicionado, entre otros factores, a la inversión, por lo que se esperaría que el crecimiento de ésta última ayude a mejorar el desempeño económico. 


\section{CUADRO 4}

MATRIZ DE TRANSICIÓN Y ESTADO ESTABLE DEL CRECIMIENTO DE LA INVERSIÓN PRIVADA.

\begin{tabular}{ccccccc}
\hline & CMA & CA & CM & CB & DEC & VPEE \\
\hline CMA & 0.193 & 0.012 & 0.000 & 0.072 & 0.723 & 0.2946 \\
CA & 0.000 & 0.000 & 0.000 & 0.250 & 0.750 & 0.0523 \\
\hline $\mathrm{CM}$ & 0.000 & 0.143 & 0.000 & 0.143 & 0.714 & 0.0337 \\
$\mathrm{CB}$ & 0.158 & 0.053 & 0.000 & 0.211 & 0.579 & 0.1045 \\
\hline $\mathrm{DEC}$ & 0.429 & 0.075 & 0.065 & 0.084 & 0.346 & 0.5148 \\
$\mathrm{VD}$ & 66 & 12 & 8 & 23 & 115 & $\mathrm{EE}=15$ \\
\hline
\end{tabular}

Nota: $\mathrm{VD}_{\mathrm{EE}}=$ vector de distribución en el estado estable, $\mathrm{VP}_{\mathrm{EE}}=$ vector de probabilidades del estado estable $\mathrm{y}$ $\mathrm{EE}=$ periodos necesarios para alcanzar el estado estable; para el resto de las variables véase el Cuadro 1. Fuente: elaboración propia.

El condicionamiento que anticipa la teoría, efectivamente, se refuerza desde esta perspectiva de análisis, aunque ahora se necesita un plazo sutilmente mayor para alcanzar el estado estable (19 periodos). Sin embargo, las condiciones de la economía mejoran en dos sentidos (lo que favorece la hipótesis). Por un lado, hay un aumento en el número de episodios de los estados que se pueden calificar como favorables (crecimiento muy alto y alto), mientras que, por otro, se observa una reducción en el número de eventos desfavorables (crecimiento bajo y decrecimiento). La reducción de situaciones en declive económico (decrecimiento) es notable, ésta va de 139 a 104, resultado que se identifica con la relación teórica entre inversión y crecimiento del producto. Es posible, entonces, reducir el tiempo de transición para alcanzar la estabilidad si dentro del sector transporte se impulsan las inversiones, lo que a su vez detonaría el crecimiento de la economía en general. 


\section{CUADRO 5}

MATRIZ DE TRANSICIÓN Y ESTADO ESTABLE DEL CRECIMIENTO CONDICIONADO (VALOR AGREGADO E INVERSIÓN PRIVADA)

\begin{tabular}{ccccccc}
\hline & CMA_INF & CA_INF & CM_INF & CB_INF & DEC_INF & VPEE \\
CMA_EC & 0.671 & 0.043 & 0.057 & 0.043 & 0.186 & 0.4219 \\
CA_EC & 0.300 & 0.200 & 0.200 & 0.000 & 0.300 & 0.0521 \\
CM_EC & 0.421 & 0.105 & 0.053 & 0.000 & 0.421 & 0.0586 \\
CB_EC & 0.222 & 0.000 & 0.056 & 0.167 & 0.556 & 0.0635 \\
DEC_EC & 0.209 & 0.043 & 0.043 & 0.086 & 0.619 & 0.4038 \\
VD & 109 & 14 & 15 & 17 & 104 & EE $=19$ \\
\hline
\end{tabular}

Nota: se usa la extensión INF para las tasas de crecimiento de la inversión privada y la extensión EC para las tasas de crecimiento del producto; para el resto de las variables véase el Cuadro 3.

Fuente: elaboración propia.

Otra forma de expresar las probabilidades de la matriz de transición de los cuadros 3, 4 y 5 se ilustra en los diagramas de transición de las figuras 1,2 y 3 , de las cuales se obtiene una perspectiva adicional de esta dinámica. En cuanto a valor agregado (Figura 1) hay tres estados que son recurrentes; es decir, existe la posibilidad de regresar a sí mismo, además, no hay un estado absorbente (cuando una vez se entra a un estado nunca se sale de éste), los diferentes estados se comunican (matriz irreducible) y son transitorios (hay accesibilidad de uno a otro).

La Figura 1 también destaca que la probabilidad de pasar de CA a DEC es muy alta, alrededor de $90 \%$, ya que no existe probabilidad de pasar de CA a CMA ni a CB. Por lo que es factible inferir que la probabilidad de mantenerse en el mismo estado es relativamente baja, excepto para decrecimiento, hecho que sugiere que salir de esa ubicación podría no ser fácil. Hay una gran volatilidad y movimiento extremo entre los estados CMA y DEC, además, se registran pocos cambios en situaciones intermedias.

En cuanto a la inversión privada (Figura 2), también se tienen tres estados recurrentes y se comparten algunas de las propiedades observadas con valor agregado (Figura 1), pero diferentemente; las probabilidades de permanecer en el mismo estado son más altas y cargadas hacia los extremos. De acuerdo con esto, la inversión resulta más volátil que el valor agregado. 
FIGURA 1

DIAGRAMA DE TRANSICIÓN ENTRE ESTADOS DEL CRECIMIENTO DEL VALOR AGREGADO.

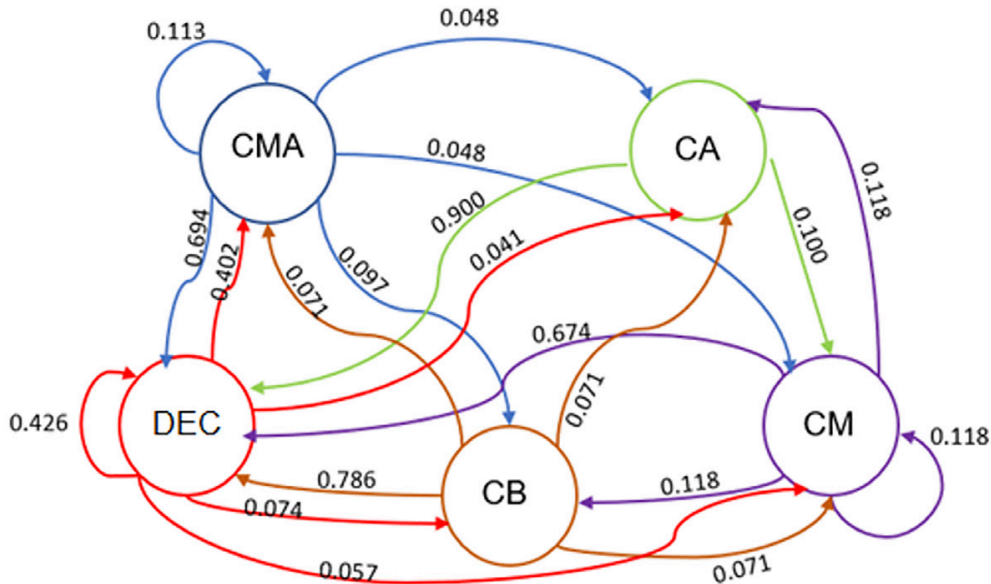

Fuente: elaboración propia.

FIGURA 2

DIAGRAMA DE TRANSICIÓN ENTRE ESTADOS DEL CRECIMIENTO DE LA INVERSIÓN PRIVADA

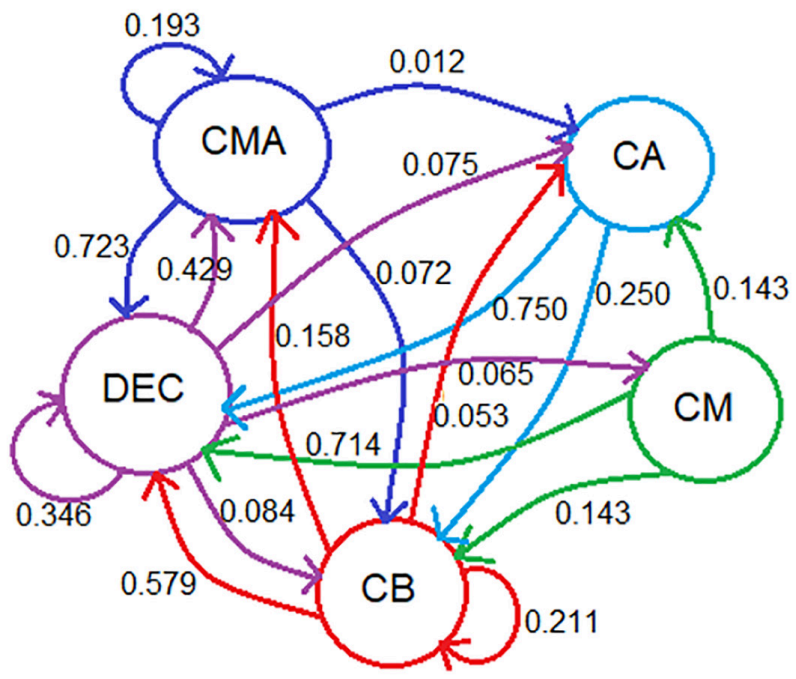

Fuente: elaboración propia. 
FIGURA 3

DIAGRAMA DE TRANSICIÓN ENTRE ESTADOS DEL CRECIMIENTO CONDICIONADO (VALOR AGREGADO E INVERSIÓN PRIVADA)

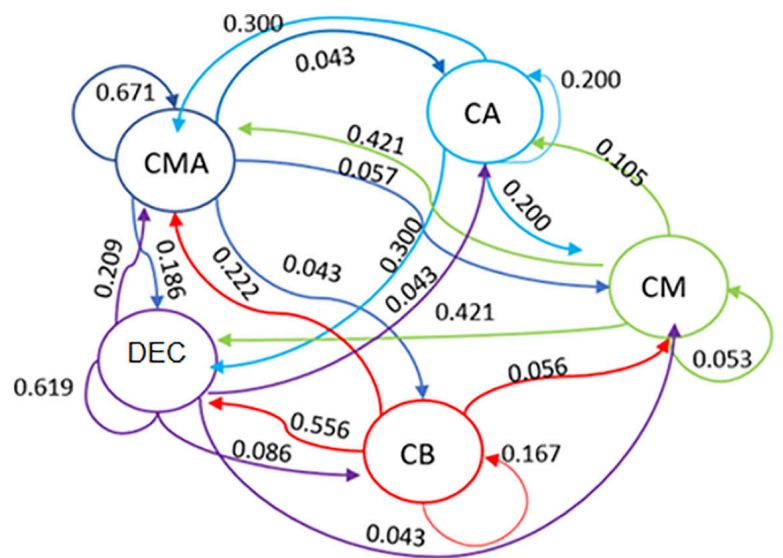

Fuente: elaboración propia.

Mientras tanto, en la Figura 3 (ejercicio condicionado) todos los estados cumplen con ser recurrentes y muestran una dinámica de transición mucho más favorable, ya que ahora es posible transitar de CA a CMA o a CM; además, la probabilidad de caer en el cuantil decrecimiento es mucho menor, aproximadamente de $30 \%$; mientras que la probabilidad de permanecer con crecimiento muy alto (CMA) al siguiente periodo es mucho más elevada, estimada ahora en 67 por ciento.

\subsection{Análisis según el grado de desarrollo socioeconómico de los estados mexicanos}

¿Cómo será la dinámica de transición en economías estatales de mayor y menor desarrollo socioeconómico? ¿Qué tipo de economía llegará más rápido al estado estable? Y, finalmente, ¿mejorarán las economías su estatus en el equilibrio? Se podría pensar que las entidades que muestran mayor desarrollo serán las más rápidas en alcanzar el estado estable o que rendirán mayores efectos desde los cambios registrados en la composición producción-inversión. Sin embargo, la revisión de estudios de secciones anteriores sugiere que los resultados no son necesariamente obvios. Debido a los rendimientos decrecientes, es posible que los movimientos de transición y los cambios en la combi- 
nación producción-inversión lleven a que las más alejadas al estado estacionario (economías de menor desarrollo) alcancen más rápido la condición de equilibrio (es decir, exhiban mayor velocidad, lo que se relacionaría con la hipótesis de la convergencia), mientras que las más cercanas (economías de mayor desarrollo) tardan más tiempo. En ambos casos, los movimientos de transición llevan a un estado estable incierto en el que la posición final sobre la dinámica del crecimiento podría ser mejor o peor que la inicial.

Para la evidencia con este otro ensayo, delimitamos el ejercicio de Markov únicamente al caso condicionado. La línea divisoria entre entidades geográficas más y menos desarrolladas se decide con base en el índice de desarrollo humano (IDH) estimado en López-Calva y Székely (2006). Ellos calculan el IDH desde variables socioeconómicas como salud, educación e ingreso, y llegan a una clasificación de entidades de alto, medio y bajo desarrollo. En el análisis que sigue se consideran únicamente los grupos con IDH alto y bajo (definidos con base en los valores medios del periodo de datos del estudio), ya que el interés radica en subrayar las diferencias de desempeño entre las economías más y menos desarrolladas. La Figura 4 reporta la geografía estatal de la distribución del índice de desarrollo humano, mientras que los cuadros 6 y 7 reportan los resultados de transición de Markov.

De manera similar a los hallazgos obtenidos con la muestra absoluta, aquí también los dos grupos de economías presentan rasgos de volatilidad elevada, ya que tanto la matriz de transición como el vector final del estado estable concentran las probabilidades más altas en los extremos. Asimismo, ambas clases de economías tardan una cantidad similar de periodos en llegar al estado estable (17 versus 18 ), por lo que en este aspecto no parecen diferir sustancialmente. Sin embargo, las economías de menor desarrollo conquistan posiciones, en el estado estable, relativamente mejores a las de mayor desarrollo. Este resultado se compara con el de otros estudios realizados entre conjuntos de países, como Barzin et al. (2018).

Por otro lado, en ambos conjuntos la dinámica de transición lleva a un estado estable en el que las probabilidades de permanecer en la situación de crecimiento muy alto se reducen notoriamente, pero esta pérdida resulta relativamente superior en las economías de mayor desarrollo. Además, hay una mejoría de la situación económica menos favorable, ya que en este caso ambos grupos reducen las probabilidades de permanecer 
en declive económico. Pero, de nueva cuenta, las economías de menor desarrollo exhiben la tasa más elevada de reducción de probabilidades, al pasar de $61 \%$, al inicio, a 33\%, al final, una caída de 28 puntos porcentuales; mientras que en las economías de mayor desarrollo esta variación alcanza apenas los 8 puntos.

\section{FIGURA 4}

\section{ENTIDADES FEDERATIVAS DE MÉXICO: GRUPOS DE ALTO Y BAJO DESARROLLO HUMANO}

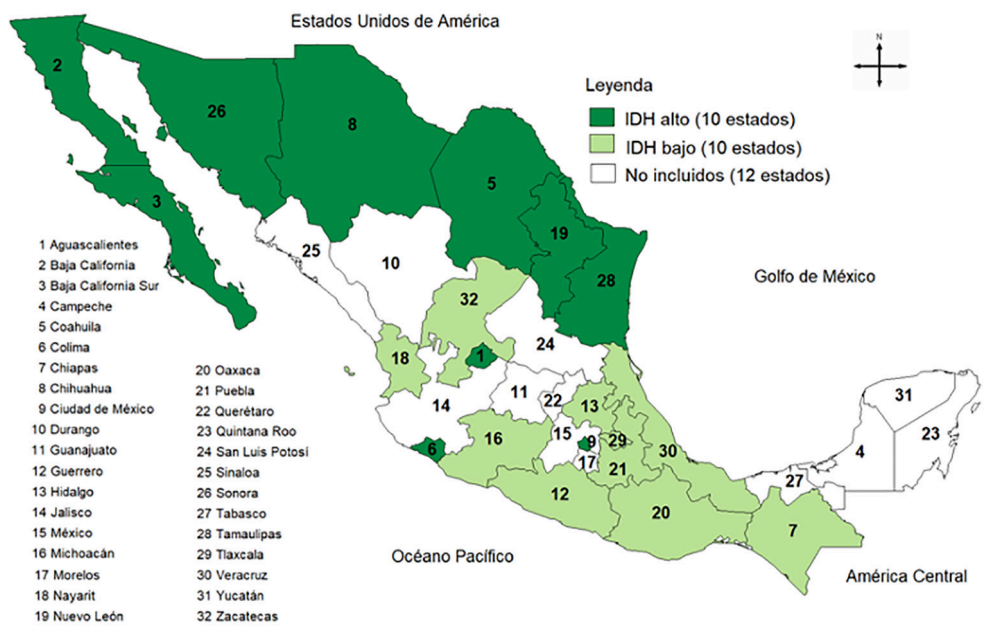

Fuente: elaborado con información sobre IDH estimado en López y Székely (2006).

En el grupo de mayor desarrollo humano (Cuadro 6) no se presentan cambios sustanciales durante el periodo de transición; sin embargo, sí se aprecian movimientos significativos en las entidades definidas como de menor desarrollo (Cuadro 7). En este segundo conjunto se observa un aumento en el número de casos de crecimiento muy alto (CMA), al pasar de 21 a 45, una variación de 24, y una disminución significativa en la cantidad de sucesos contabilizados como decrecimiento, este total va de 46 a 27 , una caída de 19 . Por tanto, se puede concluir que, de darse una fuerte inversión en infraestructura, las entidades federativas con IDH bajo mejorarían su senda del crecimiento. 


\section{CUADRO 6}

MATRIZ DE TRANSICIÓN Y ESTADO ESTABLE DEL CRECIMIENTO CONDICIONADO (ENTIDADES CON IDH MÁS ALTO)

\begin{tabular}{ccccccc}
\hline & CMA_INF & CA_INF & CM_INF & CB_INF & DEC_INF & VPEE \\
CMA_EC & 0.591 & 0.091 & 0.091 & 0.091 & 0.136 & 0.2649 \\
CA_EC & 0.000 & 0.000 & 0.000 & 0.000 & 1.000 & 0.0732 \\
\hline CM_EC & 0.167 & 0.000 & 0.000 & 0.000 & 0.833 & 0.0609 \\
CB_EC & 0.000 & 0.000 & 0.000 & 0.429 & 0.571 & 0.0851 \\
\hline DEC_EC & 0.190 & 0.095 & 0.071 & 0.048 & 0.595 & 0.5157 \\
VDINICIAL & 22 & 3 & 6 & 7 & 42 & \\
\hline VD & 22 & 6 & 5 & 7 & 42 & EE $=17$ \\
\hline
\end{tabular}

Nota: VDINICIAL = Vector de distribución inicial. Para el resto de las variables véanse las notas de los cuadros 3 y 5.

Fuente: elaboración propia.

\section{CUADRO 7}

MATRIZ DE TRANSICIÓN Y ESTADO ESTABLE DEL CRECIMIENTO CONDICIONADO (ENTIDADES CON IDH MÁS BAJO)

\begin{tabular}{ccccccc}
\hline & CMA_INF & CA_INF & CM_INF & CB_INF & DEC_INF & VPEE \\
\hline CMA_EC & 0.762 & 0.000 & 0.000 & 0.048 & 0.190 & 0.5520 \\
CA_EC & 0.250 & 0.250 & 0.500 & 0.000 & 0.000 & 0.0096 \\
\hline CM_EC & 0.600 & 0.000 & 0.200 & 0.000 & 0.200 & 0.0367 \\
CB_EC & 0.500 & 0.000 & 0.250 & 0.000 & 0.250 & 0.0695 \\
\hline DEC_EC & 0.217 & 0.022 & 0.022 & 0.130 & 0.609 & 0.3319 \\
VDINICIAL & 21 & 4 & 5 & 4 & 46 & \\
\hline VD & 45 & 1 & 3 & 6 & 27 & EE $=18$ \\
\hline
\end{tabular}

Nota: VDINICIAL = Vector de distribución inicial. Para el resto de las variables véanse notas de los cuadros 3 y 5. Fuente: elaboración propia.

\section{CONCLUSIONES}

El crecimiento económico del país durante los últimos 40 años ha sido heterogéneo y volátil. Ésta también ha sido la tónica del sector transporte, como se pudo confirmar desde el análisis de los resultados de este 
trabajo. Además, los diferentes episodios del crecimiento no exhibieron continuidad, sino que describieron trayectorias de altibajos, aumentos y caídas repentinas. Esto no es más que el reflejo de los vaivenes macroeconómicos acentuados por la configuración de eventos adversos, como las crisis económicas y monetarias, el elevado desempleo, la devaluación de la moneda y los periodos de alta inflación. Como resultado, hubo mucha incertidumbre y sensibilidad en las decisiones de inversión del sector privado y, por tanto, en el desempeño relacionado con el crecimiento de la producción, medida desde el valor agregado.

El análisis delimitado al sector transporte con el modelo de Markov sugiere que, dadas las características económicas que presenta esta actividad, tomará varios años lograr que se controle la volatilidad en el crecimiento; sin embargo, una vez alcanzada, las condiciones serán mejores que al inicio. De no aplicarse las políticas necesarias, la volatilidad que caracteriza al crecimiento económico mexicano, en particular en el transporte, difícilmente permitirá agilizar la salida de esta situación de "trampa del crecimiento", ya que resulta complicado mejorar su desempeño. Al menos en el corto plazo, la ausencia de intervención pública o privada, o la canalización insuficiente de inversiones, puede ir postergando el estado estable.

Los resultados tienen algunas implicaciones para el diseño de políticas de inversión privada en infraestructura, en general, y para programas de política regional, en particular. De acuerdo con esto, una política de infraestructura del transporte nacional debe tener una perspectiva regional que considere su diversidad, así como la variedad de formas con la que los estados de la república afrontan las dificultades para impulsar el crecimiento. En particular, los resultados indican la necesidad de diseñar políticas regionales especialmente dirigidas a resolver las trampas a las que puede conducir un proceso de transición en el largo plazo. Por ejemplo, se pueden priorizar inversiones clave que detonen el crecimiento en regiones más atrasadas, sin descuidar los proyectos de las regiones de mayor avance. De acuerdo con los hallazgos, invertir en más y mejores carreteras, puentes e infraestructura, en general, rinde frutos en el crecimiento y en el bienestar de la población, pero deben observar un carácter de continuidad, apuntalados con estrictos programas de seguimiento para no descuidar su eficaz funcionamiento. Esta clase de políticas regionales podría abrir la posibilidad de cambiar 
las condiciones de largo plazo hacia uno que armonice más con crecimiento económico continuo.

Una limitación del enfoque de Markov es que requiere que las observaciones sean estocásticamente independientes (Bickenbach y Bode, 2003); sin embargo, la dinámica de inversión y producción podría estar afectada por el espacio geográfico donde se llevan a cabo, por lo que una avenida de investigación recomendable es abordar la posibilidad de que existan efectos derrame desde dependencia espacial en la matriz de probabilidades, al igual que se hace en Torres et al. (2017) con el análisis de la distribución espacial de la inversión extranjera entre los estados mexicanos.

\section{BIBLIOGRAFÍA}

Aschauer, David Alan (1990), “Why Is Infrastructure Important?”, Federal Reserve Bank of Boston, Conference Series, 21-50.

Barzin, Samira, Sabine D’Costa y Daniel J. Graham (2018), “A Pseudo-Panel Approach to Estimate Dynamic Effects of Road Infrastructure on Firm Performance in a Developing Country Context", Regional Science and Urban Economics, 70, pp. 20-34.

Bickenbach, Frank y Eckardt Bode (2003), "Evaluating the Markov Property in Studies of Economic Convergence", International Regional Science Review, 26(3), pp. 363-392.

Chen, Zhenhua y Kingsley E. Haynes (2015), "Regional Impact of Public Transportation Infrastructure: A Spatial Panel Assessment of the U.S. Northeast Megaregion”, Economic Development Quarterly, 29(3), pp. 275-291.

Cohen, Jeffrey P. y Catherine J. Morrison Paul (2003), "Airport Infrastructure Spillovers in a Network System", Journal of Urban Economics, 54(3), pp. 459-473.

Cohen, Jeffrey P. y Catherine J. Morrison Paul (2004), "Public Infrastructure Investment, Interstate Spatial Spillovers, and Manufacturing Costs", Review of Economics and Statistics, 86(2), pp. 551-560.

Dasgupta, Dipankar (1999), "Growth Versus Welfare in a Model of Nonrival Infrastructure", Journal of Development Economics, 58(2), pp. 359-385.

Deichmann, Uwe, Marianne Fay, Jun Koo y Somik V. Lall, (2004), "Economic Structure, Productivity, and Infrastructure Quality in Southern Mexico", The Annals of Regional Science, 38, pp. 361-385.

Di Giacinto, V., G. Micucci y P. Montanaro (2012), "Network Effects of Public Transport Infrastructure: Evidence on Italian Regions", Papers in Regional Science, 91(3), pp. 515-541.

Ding, C. (2013), "Transport Development, Regional Concentration and Economic Growth", Urban Studies, 50(2), pp. 312-328. 
Ding, Lei, Haynes, E. Kingsley y Yanchun Liu (2008), “Telecommunications Infrastructure and Regional Income Convergence in China: Panel Data Approaches", The Annals of Regional Science, 42, pp. 843-861.

Durán-Fernández, Roberto y Georgina Santos (2014a), "Regional Convergence, Road Infrastructure, and Industrial Diversity in Mexico", Research in Transportation Economics, 46, pp. 103-110.

Durán-Fernández, Roberto y Georgina Santos, (2014b), "Road Infrastructure Spillovers on the Manufacturing Sector in Mexico", Research in Transportation Economics, 46, pp. 17-29.

Eslahchi, Changiz y Fateme Movahedi (2012), "Calculation of Transition Probabilities in the Birth and Death Markov Process in the Epidemic Model", Mathematical and Computer Modeling, 55, pp. 810-815.

Fingleton, Bernard (1997), "Specification and Testing of Markov Chain Models: An Application to Convergence in the European Union", Oxford Bulletin of Economics and Statistics, 59(3), pp. 385-403.

Fingleton, Bernard y Nikodem Szumilo (2019), "Simulating the Impact of Transport Infrastructure Investment on Wages: A Dynamic Spatial Panel Model Approach", Regional Science and Urban Economics, 75, pp. 148-164.

Fuentes, Noe Aron (2003), "Desigualdades regionales en México: los efectos de la infraestructura", Comercio Exterior, 53(11), pp. 1002-1010.

Fuentes, Noe Aron y J. Eduardo Mendoza Cota (2003), "Infraestructura pública y convergencia regional en México, 1980-1998”, Comercio Exterior, 53(2), pp. 178-187.

German-Soto, Vicente y Héctor A. Barajas Bustillos (2014), "The Nexus between Infrastructure Investment and Economic Growth in the Mexican Urban Areas", Modern Economy, 5(13), pp. 1208-1220.

Hillier S. Frederick y J. Gerald Lieberman (2010), Introducción a la investigación de operaciones, McGraw-Hill, México.

$\mathrm{Hu}$, Angang y Shenglong Liu (2010), "Transportation, Economic Growth and Spillover Effects: The Conclusion Based on the Spatial Econometric Model", Frontiers of Economics in China, 5(2), pp. 169-186.

Kang, Wei y Sergio J. Rey (2018), “Conditional and Joint Tests for Spatial Effects in Discrete Markov Chain Models of Regional Income Distribution Dynamics", The Annals of Regional Science, 61(1), pp. 73-93.

Khadaroo, J. y B. Seetanah (2008), "Transport and Economic Performance: The case of Mauritus", Journal of Transport Economics and Policy, 42(2), pp. 255-267.

Kyriacou, Andreas P., Leonel Muinelo-Gallo y Oriol Roca-Sagalés (2019), “The Efficiency of Transport Infrastructure Investment and the Role of Government Quality: An Empirical Analysis”, Transport Policy, 74, pp. 93-102.

Lall, Somik V. (2007), "Infrastructure and Regional Economic Growth, Growth Dynamics and Policy Relevance for India”, The Annals of Regional Science, 41, pp. 581-599.

Lauchlin, C. (1981), “Allyn Young y el desarrollo de la teoría del crecimiento", 
Cuadernos de Economía, 13(18-19), pp. 207-224.

Le Gallo, Julie (2004), "Space-Time Analysis of GDP Disparities Among European Regions: A Markov Chains Approach”, International Regional Science Review, 27(2), pp. 138-163.

LeSage, James P. y Wolfang Polasek (2008), "Incorporating Transportation Network Structure in Spatial Econometric Models of Commodity Flows", Spatial Economic Analysis, 3(2), pp. 225-245.

López-Calva, Luis F. y Miguel Székely (2006), Medición del desarrollo humano en México, FCE, México.

Maibach, Edward W., Lorien C. Abroms y Mark Marosits (2007), "Communication and Marketing as Tools to Cultivate the Public's Health: A Proposed 'People and Places' Framewrok', BMC Public Health, 7, article 88.

Maparu, Tuhin Subhra y Tarak Nath Mazumber (2017), "Transport Infrastructure, Economic Development and Urbanization in India (1990-2011): Is There any Causal Relationship?", Transportation Research Part A: Policy and Practice, 100, pp. 319-336.

Munnell, Alicia H. (1990), "How Does Public Infrastructure Effect Regional Economic Performance?", New England Economic Review, septiembre, pp. 11-33.

Nombela, Gustavo (2005), "Infraestructuras de transporte y productividad", Presupuesto y Gasto Público, 39, pp. 191-215.

OECD (2002), Impact of Transport Infrastructure Investment on Regional Development, Paris.

Pereira, Alfredo M. y Jorge M. Andraz (2006), "Public Investment in Transportation Infrastructures and Regional Asymmetries in Portugal", The Annals of Regional Science, 40, pp. 803-817.

Quah, Danny T. (1993), "Empirical Cross-Section Dynamics in Economic Growth", European Economic Review, 37(2 y 3), pp. 426-434.

Sánchez-Robles, Blanca (1998), "Infrastructure Investment and Growth: Some Empirical Evidence”, Contemporary Economic Policy, 16(1), pp. 98-108.

Timilsina, Govinda, Gal Hochman y Ze Song (2020), "Infrastructure, Economic Growth, and Poverty", Policy Research, Working Paper núm. 9258.

Torres, V. H., M. Polanco Gaytán y M. A. Tinoco Zermeño (2017), "Dinámica de la inversión extranjera directa en los estados de México: un análisis de cadenas de Markov espaciales", Contaduría y Administración, 62, pp. 141162.

Walke, Adam G., Thomas M. Fullerton Jr., Martha Patricia Barraza de Anda y Lisbeily Domínguez Ruvalcaba (2015), “An Empirical Analysis of Education, Infrastructure, and Regional Growth in Mexico", Journal of Economics and Development Studies, 3(4), pp. 1-12. 